\title{
NUCLEUS PULPOSUS CELL-MATRIX INTERACTIONS WITH LAMININS
}

\author{
C.L. Gilchrist ${ }^{1}$, A.T. Francisco ${ }^{1}$, G.E. Plopper ${ }^{3}$, J. Chen ${ }^{2}$ and L.A. Setton ${ }^{1,2 *}$ \\ ${ }^{1}$ Department of Biomedical Engineering, Duke University, Durham, NC, USA \\ ${ }^{2}$ Department of Orthopaedic Surgery, Duke University Medical Center, Durham, NC, USA \\ ${ }^{3}$ Department of Biomedical Engineering, Rensselaer Polytechnic Institute, Troy, NY, USA
}

\begin{abstract}
The cells of the nucleus pulposus (NP) region of the intervertebral disc play a critical role in this tissue's generation and maintenance, and alterations in NP cell viability, metabolism, and phenotype with aging may be key contributors to progressive disc degeneration. Relatively little is understood about the phenotype of NP cells, including their cell-matrix interactions which may modulate phenotype and survival. Our previous work has identified strong and region-specific expression of laminins and laminin cell-surface receptors in immature NP tissues, suggesting laminin cell-matrix interactions are uniquely important to the biology of NP cells. Whether these observed tissue-level laminin expression patterns reflect functional adhesion behaviors for these cells is not known. In this study, we examined NP cell-matrix interactions with specific matrix ligands, including various laminin isoforms, using quantitative assays of cell attachment, spreading, and adhesion strength. NP cells were found to attach in higher numbers and exhibited rapid cell spreading and higher resistance to detachment force on two laminin isoforms (LM-511,LM-332) identified to be uniquely expressed in the NP region, as compared to another laminin isoform (LM111) and several other matrix ligands (collagen, fibronectin). Additionally, NP cells were found to attach in higher numbers to laminins as compared to cells isolated from the disc's annulus fibrosus region. These findings confirm that laminin and laminin receptor expression documented in NP tissues translates into unique functional NP cell adhesion behaviors that may be useful tools for in vitro cell culture and biomaterials that support NP cells.
\end{abstract}

Keywords: Intervertebral disc, nucleus pulposus, annulus fibrosus, extracellular matrix, laminin, collagen, fibronectin, cell adhesion, cell morphology, notochordal cell.

\footnotetext{
*Address for correspondence:

Lori A. Setton

Department of Biomedical Engineering

136 Hudson Hall, Box 90281

Durham, NC 27708, USA
}

E-mail: lori.setton@duke.edu

\section{Introduction}

The human intervertebral disc (IVD) is a dynamic tissue which undergoes significant changes in matrix structure, composition, and cell population during growth, aging, and with disc degeneration. The most dramatic changes in the IVD occur within the centrally located nucleus pulposus (NP) region, which transitions from a fluid-like gel to more cartilage-like tissue within the first decades of life, and with aging or degeneration exhibits further decrease in proteoglycan and water content (Gower and Pedrini, 1969; Buckwalter, 1995). One documented change in the human NP that appears to coincide with, or precede, the tissue's compositional and structural changes is the loss of, or phenotypic change to, the population of notochordallyderived NP cells (Trout et al., 1982b; Boos et al., 2002; Roberts et al., 2006; Minogue et al., 2010; Risbud et al., 2010). These cells are remnants from the embryonic notochord that are retained within the developing NP (Goto and Uhthoff, 1986; Cotten et al., 1994; Rufai et al., 1995; Choi et al., 2008), and are characterized by their distinct morphological appearance, with very large sizes, containing high numbers of vacuoles, and exhibiting strong cell-cell interactions (Trout et al., 1982b; Hunter et al., 2003b). In addition, these NP cells may express mRNA and protein markers that distinguish them from other cell types within the intervertebral disc (Fujita et al., 2005; Chen et al., 2006; Lee et al., 2007; Sakai et al., 2009; Minogue et al., 2010; Risbud et al., 2010). In addition to their role in NP matrix biosynthesis (Bayliss et al., 1988; Cappello et al., 2006; Erwin and Inman, 2006), these cells have been shown to secrete soluble factors that stimulate matrix synthesis and cell proliferation in other cell types in the disc (Aguiar et al., 1999; Boyd et al., 2004; Erwin and Inman, 2006). The biological behaviors and unique phenotype of immature NP cells are of interest for understanding their role in producing and maintaining a hydrated, mechanically-functional NP tissue (Aguiar et al., 1999; Hunter et al., 2003a; Chen et al., 2006; Erwin et al., 2006; Korecki et al., 2010), and for understanding factors that may promote retention of cells and extracellular matrix (ECM) synthesis in the maturing or aging IVD.

Cell-matrix interactions are known to modulate a wide range of cell behaviors, with particular ECM ligandreceptor (e.g., integrins (Hynes, 1992)) adhesions eliciting specific cellular responses. Laminins are key cell-adhesive ligands in a variety of tissues, formed as heterotrimeric proteins composed of $\alpha, \beta$, and $\gamma$ chains that comprise 15 known laminin isotypes (Colognato and Yurchenco, 2000; Miner and Yurchenco, 2004). Specific laminin isoforms are known to modulate cell differentiation, phenotype, 
survival, and organization in a variety of cells and tissues including epithelia, bone, and neural tissues, as well as in embryonic and mesenchymal stem cells (Gu et al., 2002; Miner and Yurchenco, 2004; Klaffky et al., 2006; Klees et al., 2007; Rodin et al., 2010). Previous studies in our laboratory have identified NP-specific expression of select laminin isoforms (LM-511) and receptors (integrins $\alpha 6$ and $\beta 4, \mathrm{CD} 239)$ in immature IVD tissues and isolated cells (Nettles et al., 2004; Gilchrist et al., 2007; Chen et al., 2009). Additionally, immature NP cells were found to adhere readily to laminin isoform LM-111, with adhesion of the larger, vacuolated NP cells found to be mediated via the $\alpha 6$ integrin subunit (Gilchrist et al., 2007). Whether specific laminins promote adhesion of immature NP cells relative to other ECM ligands, however, is not yet known. Knowledge of preferred ECM ligands for NP cells may advance the development of NP cellsupportive biomaterials that promote or maintain an NP cell phenotype, and may elucidate cell-ligand interactions specific to the NP that have potential to modulate biological behaviors of differentiation, phenotypic maintenance and cell survival.

The objective of this study was to quantify immature NP cell adhesion behaviors on specific ECM ligands found in the IVD. Based on the known isoforms of laminin identified in NP tissues, as well as the novel identification of laminin LM-332 reported in this study, we hypothesized that immature NP cells would preferentially adhere to laminins as compared to several other ECM ligands found in the IVD, and that laminin isoforms identified as highly expressed in the NP region (LM-511, LM-332) would promote the greatest NP cell-matrix interactions (attachment, spreading, resistance to applied detachment forces). Additionally, we hypothesized that immature NP cells would attach more readily to laminins as compared to cells from the adjacent annulus fibrosus (AF) region, where expression of laminins in situ appears limited. To investigate these hypotheses, quantitative assays of cell attachment, cell spreading, and mechanical cell attachment strength were used to compare IVD cell interactions with specific ECM constituents.

\section{Materials and Methods}

\section{Immunohistochemistry}

Immature porcine (3-6 months old, from local abattoir) and rat (1 month-old) lumbar IVD tissues were embedded in OCT medium (Sakura Finetek, Torrance, CA, USA), flash-frozen in liquid nitrogen, and stored at $-80{ }^{\circ} \mathrm{C}$ until cryosectioning. Frozen sections $(10 \mu \mathrm{m})$ were fixed (4 $\%$ formaldehyde), permeabilized ( $0.2 \%$ Triton X-100), blocked (3.75\% BSA, $5 \%$ rabbit serum), and incubated with primary antibody specific for the laminin $\gamma 2$ chain ( $2 \mathrm{~h}$ at room temperature; goat polyclonal C-20, Santa Cruz Biotechnology, Santa Cruz, CA, USA (Robbins et al., 2001)), which uniquely identifies the LM-332 isoform (Colognato and Yurchenco, 2000). Samples were fluorescently labeled with secondary antibody (30 min at room temperature; Alexa 488, Invitrogen, Carlsbad, CA, USA) and propidium iodide (to label cell nuclei,
Sigma-Aldrich, St. Louis, MO, USA). Negative controls included samples incubated with secondary antibodies only. Stained tissue sections ( $n=3$ IVD samples for each species, multiple sections per sample) were imaged using a laser scanning confocal microscope (Zeiss LSM 510, 20X 0.5NA objective; Carl Zeiss, Oberkochen, Germany).

\section{Cell isolation and culture}

Lumbar spines were obtained from skeletally immature pigs (3-6 months old, from local abattoir) within $8 \mathrm{~h}$ postsacrifice. IVDs from this source have been shown to be rich in larger, highly vacuolated NP cells (Chen et al., 2006; Gilchrist et al., 2007). Cells were isolated from the NP and AF regions of lumbar IVDs via pronase-collagenase enzymatic digestion ((Chen et al., 2002); tissue pooled from lumbar IVDs of one animal to generate a single cell population for each region (NP, AF)) and resuspended in culture media (Ham's F-12 media (Gibco, Invitrogen) supplemented with $5 \%$ FBS (Hyclone, Thermo Scientific, Rockford, IL, USA), 10mM HEPES (Gibco), 100 U/mL penicillin (Gibco) and $100 \mathrm{mg} / \mathrm{mL}$ streptomycin (Gibco)). Isolated cells were cultured in subconfluent monolayers $\left(50,000\right.$ cells $\left./ \mathrm{cm}^{2}\right)$ on $0.1 \%$ gelatin-coated (Sigma-Aldrich) T75 tissue culture flasks and used prior to passaging within 3-7 d; this protocol was adopted to allow for optimal reexpression of any cell surface receptors potentially affected by the enzymatic cell isolation process (Loeser et al., 1993; Gilchrist et al., 2007). For some experiments, cells were used immediately following isolation (in contrast to the monolayer culture protocol) and noted as "freshly isolated".

\section{Cell adhesion experiments}

To create ECM substrates presenting specific matrix ligands for cell attachment, 96-well culture plates (Corning Costar, Lowell, MA, USA) were coated (via adsorption, overnight at $4{ }^{\circ} \mathrm{C}$ ) with one of the following ECM proteins diluted in phosphate buffered saline (PBS): chicken type II collagen (40 $\mu \mathrm{g} / \mathrm{mL}$, Sigma-Aldrich), human plasma fibronectin (FN, $40 \mu \mathrm{g} / \mathrm{mL}$, Gibco), mouse LM-111 (mouse EHS laminin, $40 \mu \mathrm{g} / \mathrm{mL}$, Sigma-Aldrich), LM511 (human placental laminin, Sigma-Aldrich, $20 \mu \mathrm{g} /$ $\mathrm{mL}$; this commercial preparation has been determined to consist primarily of laminin-511 (Wondimu et al., 2006)); coating solution concentrations were chosen to insure saturation of adsorbed protein, which maximized NP cell adhesion in preliminary studies. In addition to these purified proteins, a substrate rich in LM-332 (which is not commercially available and not readily purified) was created by incubating culture wells with conditioned medium collected from rat carcinoma cell line 804G (as described below). All coated wells were blocked with 3.75 $\%$ bovine serum albumin (BSA, Gibco) for $3 \mathrm{~h}$ at $37^{\circ} \mathrm{C}$, and wells without matrix protein (BSA-blocked only) were used to identify non-specific cell attachment.

The rat carcinoma cell line 804G (Plopper et al., 1998) was used to generate LM-332-containing conditioned media (LM-332CM). 804G cells were first grown to semiconfluence in growth media (Dulbecco's Modified Eagle's Medium (DMEM) with $10 \%$ FBS, 10 mM HEPES, and 2 $\mathrm{mM}$ L-glutamine). Culture media was then replaced with 
A)
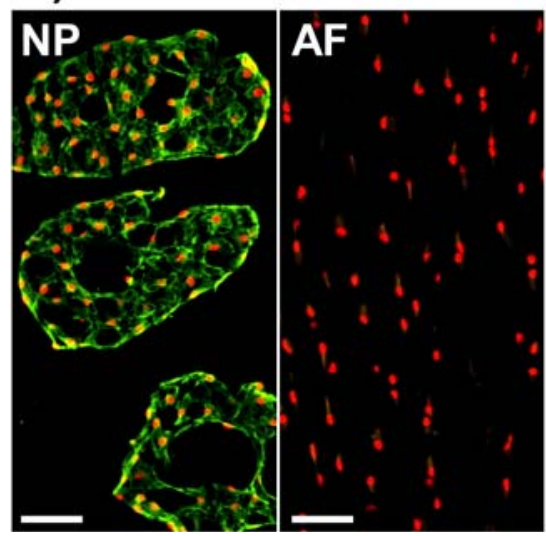

B)

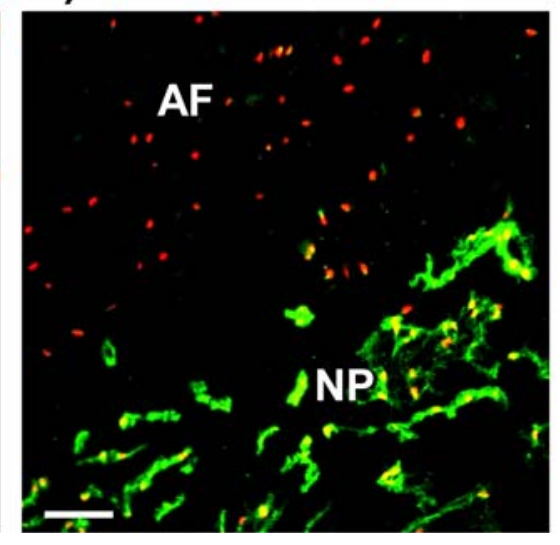

C)

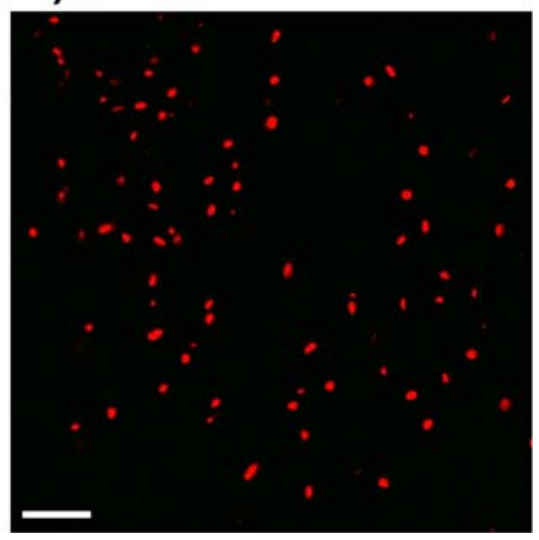

Fig. 1. Expression of the laminin $\gamma 2$ chain in IVD tissues. The expression of the laminin $\gamma 2$ chain, which uniquely identifies the LM-332 laminin isoform, was assessed in immature (A) porcine and (B) rat IVD tissues by means of immunohistochemistry, with a representative negative control (rat) shown in (C). The $\gamma 2$ chain was found to be highly expressed in the NP region of both species, with NP cell clusters exhibiting intense, cell-associated laminin staining. In contrast, only occasional, faint cell-associated staining was detected in the AF region of either species (green: laminin $\gamma 2$, red: cell nuclei). Scale bars $=50 \mu \mathrm{m}$.

serum-free media (same as formulation above, without serum) and cells cultured for an additional $3 \mathrm{~d}$. Conditioned medium was collected, clarified $(15,000 \mathrm{~g}, 5 \mathrm{~min})$, and the supernatant stored at $-20^{\circ} \mathrm{C}$ until use. To create LM-332CM substrates, wells were coated with undiluted conditioned media overnight $\left(4^{\circ} \mathrm{C}\right)$, washed with PBS, and blocked with BSA as described above. The presence of LM-332 on well surfaces was confirmed using an anti-LM-332 antibody (clone FM3 (Plopper et al., 1996)).

NP cell adhesion experiments were performed initially with freshly isolated cells to test for an effect of NP cell adhesiveness to laminin isoforms immediately upon isolation, in comparison to conventional plate coatings (e.g., collagen). For all cell attachment experiments, cells were resuspended in serum-free F12 media and seeded on extracellular matrix (ECM) substrates (10,000 cells/well). Following a specific attachment period $\left(0.5\right.$ or $4 \mathrm{~h}, 37^{\circ} \mathrm{C}$, $5 \% \mathrm{CO}_{2}$ ), wells were gently rinsed twice with serumfree media to remove unattached cells. Remaining cells were fixed ( $4 \%$ formaldehyde), cell nuclei were labeled (Hoechst 33342, Sigma-Aldrich), and cells were imaged via fluorescence microscopy (1 image/well, 10X objective, Zeiss Axiovert S100). Differences in attachment numbers amongst ECM substrates were detected using ANOVA with Tukey's post hoc test ( $p<0.05, n=4$ independent experiments from separate cell isolations, 4 replicate wells per substrate condition).

Adhesion experiments were also performed with cells from the NP and adjacent AF for assessment of differences in cell-laminin ligand interactions amongst these cell types. Cells from monolayer culture (cultured 3-7 d) were used for these studies in order to promote re-expression of NP and AF cell surface receptors capable of participating in cell-ligand interactions (Loeser, 1993; Gilchrist et al., 2007). Cells were detached from the culture flask with $0.025 \%$ trypsin/EDTA (Cambrex, East Rutherford, NJ, USA), followed by washing with soybean trypsin inhibitor
( $1 \mathrm{mg} / \mathrm{mL}$, Sigma). Cells were resuspended in serum-free F12 media and seeded on laminin only substrates $(10,000$ cells/well; LM-111, LM-511, LM-332CM); following a 1 $\mathrm{h}$ attachment period $\left(37^{\circ} \mathrm{C}, 5 \% \mathrm{CO}_{2}\right)$, wells were gently rinsed twice with serum-free media, and cell numbers were determined as described above for NP cell adhesion experiments. Differences amongst cell types and laminin substrates were analyzed by means of two-factor analysis of variance (ANOVA), with Tukey's post hoc test ( $p<$ $0.05, n=6$ independent experiments from separate cell isolations, 4 replicate wells/condition).

\section{Cell spreading and morphology}

To examine the effect of ECM ligand on NP cell morphology and spreading dynamics, NP cells were seeded onto ECM substrates and cell spread areas (2D projections) were measured over time. NP cells from monolayer culture (3-7 d) were detached from the culture surface, resuspended in serum-free culture media, and seeded (5,000 cells/well) onto 96-well plates coated with type II collagen, fibronectin, LM-111, LM-511 or LM$332 \mathrm{CM}$ as described above at the coating concentrations described above. Cells were allowed to attach for periods of 1-4 h, then fixed in $4 \%$ formaldehyde (15 min at room temperature). Cell actin cytoskeleton (used to define cell boundary) and cell nuclei were labeled by permeabilizing $(0.2 \%$ Triton X-100) and incubating with Alexa Fluor 488 phalloidin (1:250 in PBS) and propidium iodide (200 $\mu \mathrm{g} / \mathrm{mL})$. Fluorescent images of cells were obtained using a confocal microscope (Zeiss LSM 510, Plan-Neofluar $20 \mathrm{X}$ objective), with projected areas and perimeters of individual cells measured from acquired images (Nikon NIS-Elements BR, Melville, NY). As a measure of cell morphology, a dimensionless cell shape factor SF was computed for each cell (Engler et al., 2004), defined as $S F=4 \pi A / p^{2}$, where $A=$ projected cell area and $p=$ cell perimeter. A total of three $(n=3$ experiments from separate 


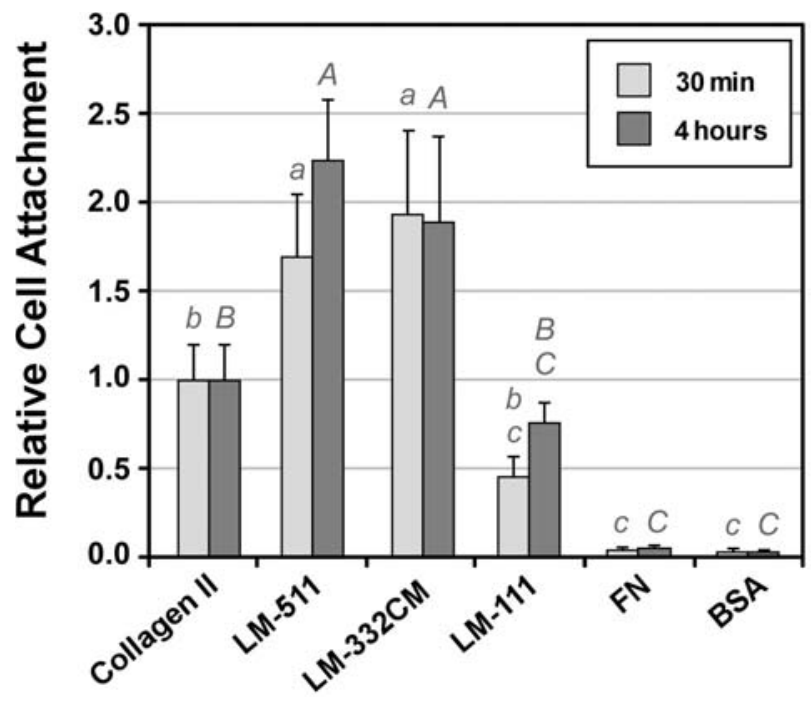

Fig. 2. Relative attachment of freshly-isolated NP cells to ECM substrates. Following enzymatic isolation, NP cells were allowed to attach to ECM substrates for either $30 \mathrm{~min}$ or $4 \mathrm{~h}$, with cell numbers normalized to those attaching to collagen substrates at each time point. Conditions labeled with different letters were statistically different (1-factor ANOVA, $p<0.05$ ); comparisons between time points were not analyzed; lower case $=$ differences at $30 \mathrm{~min}$ amongst substrates, upper case $=$ at $4 \mathrm{~h}$.

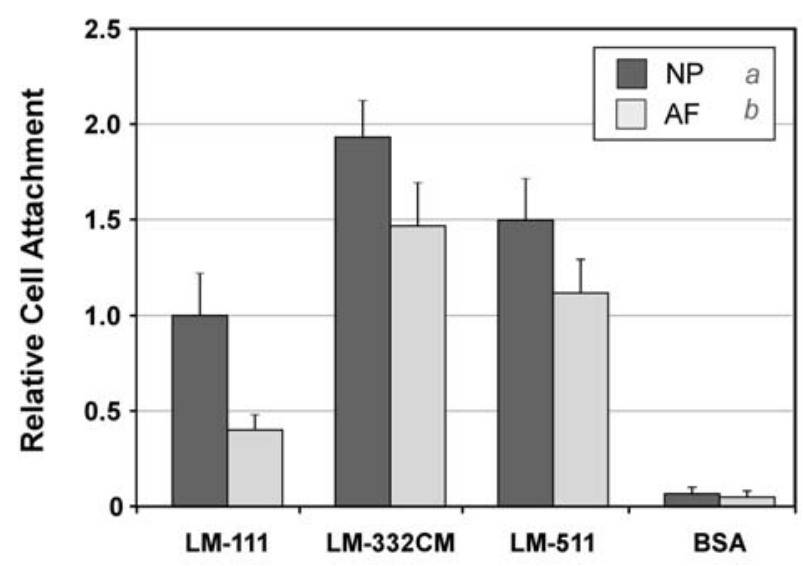

Fig. 3. Adhesion of NP and AF cells to laminin isoforms. Cells isolated from porcine NP and AF regions of porcine IVDs were allowed to attach to laminin ECM substrates for $1 \mathrm{~h}$ in order to test for differences in binding patterns between NP and AF cells. NP cells were found to attach to laminin substrates at significantly higher numbers compared to AF cells (cell attachment levels normalized to NP cells on LM-111; $p<0.01$; two-factor ANOVA (cell type, ECM substrate), mean \pm SEM). Differences between cell types for specific laminin isoforms were not assessed, as cell type-substrate interaction was not significant $(p=0.06)$. cell isolations) cell-spreading experiments were performed, with a minimum of 100 cells analyzed for each condition (ECM substrate, time point). A two-way ANOVA (ECM substrate, time) was performed to analyze cell spreading and shape factor experiments, using Tukey's post hoc test to detect differences between substrates and with time ( $p$ $<0.05$ significant).

\section{Cell attachment strength}

A centrifugal cell adhesion assay (Lotz et al., 1989; Reyes and Garcia, 2003) was performed to assess the relative attachment strength on different ECM substrates (fibronectin, type II collagen LM-111, LM-511, LM$332 \mathrm{CM}$ at concentrations given above). NP cells in monolayer culture (3-7 d) were incubated with calcein-AM (Invitrogen) diluted in $2 \mathrm{mM}$ PBS-dextrose for $20 \mathrm{~min}$ at 37 ${ }^{\circ} \mathrm{C}$. Fluorescently-labeled cells were detached from culture flasks, resuspended in PBS-dextrose, and seeded at 10,000 cells/well onto ECM-coated 96-well plates (Corning Costar, black opaque). Cells were incubated for $90 \mathrm{~min}$ at room temperature to allow for cell attachment. After one gentle wash with PBS-dextrose, a pre-spin reading was performed with a fluorescence plate reader (Tecan Genios, Mannendorf, Switzerland) to quantify cell numbers in each well prior to application of a defined centrifugal detachment force. Plates were then covered with sealing tape, inverted, and spun in a benchtop centrifuge at either $200 \mathrm{~g}$ or $600 \mathrm{~g}$. Wells were refilled with PBS, washed once gently to remove any detached cells, and a post-spin fluorescence reading was obtained.

Pre-spin and post-spin values for each well were normalized by subtracting out the background signal from negative controls of BSA blocked wells (no seeded cells). The fraction of adherent cells, $f$, was then computed for each well as: $f=R F U_{\text {post }} / R F U_{\text {pre }}$, where RFU is relative fluorescence units as measured by the plate reader. Control wells containing known amounts of cells confirmed that cell fluorescence signal was linearly related to cell number, with $\mathrm{R}^{2}>0.99$ for all experiments. Three independent experiments ( $n=3$ separate cell isolations, 6 wells/ condition) were performed, with differences amongst substrates detected by means of one-factor ANOVA with Tukey's post-hoc analysis.

\section{Results}

\section{LM-332 expression in the IVD}

Previous studies (Chen et al., 2009) identified strong expression of the LM-511/-521 isoforms (as identified by the $\alpha 5$ laminin subunit) in the immature NP, but not AF, region of IVD tissues. We investigated whether another cell-adhesive laminin isoform, LM-332, might also be expressed in IVD tissues. The laminin $\gamma 2$ chain, which uniquely identifies the LM-332 isoform, was found to be highly expressed in the NP region of both porcine and rat intervertebral disc tissues, as shown in Fig. 1. NP cells (arranged in cell clusters as previously documented (Hunter et al., 2003b; Trout et al., 1982b)) exhibited intense cell-associated staining in all NP tissue sections from both species. In contrast, AF tissues exhibited low levels of staining for LM-332, with faint (but consistent) 

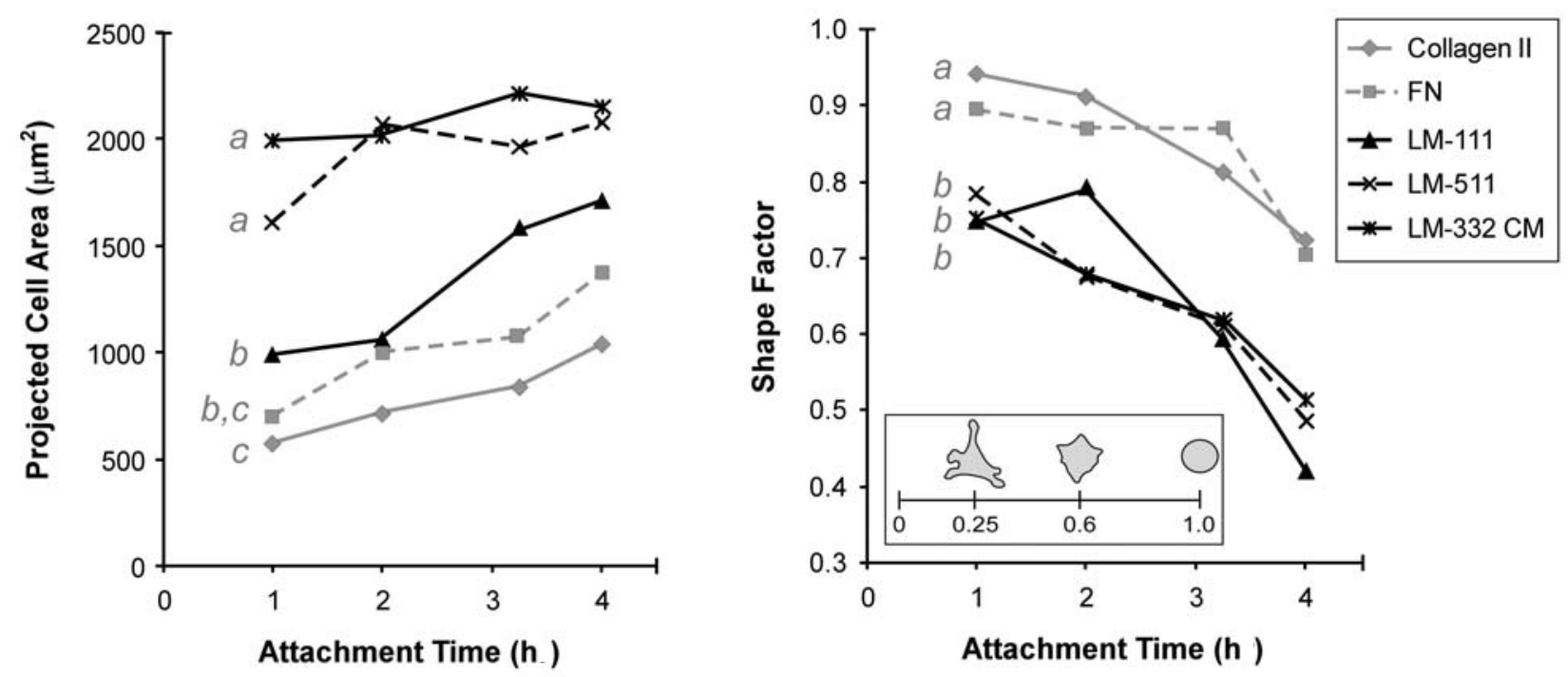

Fig. 4. NP cell spreading dynamics and shape factors on ECM substrates. Cell projected areas and non-dimensional shape factors were analyzed during NP cell attachment to ECM substrates over time $(n=3$ independent experiments, $>100$ cells per condition/time point). Shape factor inset (right) illustrates representative cell morphologies and corresponding shape factors. Significant effects of substrate and time were detected for both cell area and shape (two-way ANOVA, $p<0.005$ ); substrates not labeled with same letter were statistically different.

cell-associated staining in the rat AF, and very low or completely absent staining in the pig AF. In rat IVD tissues where the smaller disc could be sectioned intact, LM-332 expression clearly demarcated the boundary between NP and AF regions (Fig. 1B). This differential staining pattern between NP and AF regions is similar to that observed for the LM-511 isoform (as previously identified (Chen et al., 2009)) and for cell-surface receptors known to bind the LM-511 and LM-332 isoforms ( $\alpha 6$ and $\beta 4$ integrins, CD239 (Nettles et al., 2004; Gilchrist et al., 2007; Chen et al., 2009), but differs from findings for the laminin LM-111 isoform, which was previously found to be only weakly expressed in immature IVD tissues, and not specific to the NP region (Chen et al., 2009).

\section{NP cell adhesion to laminin isoforms}

Since freshly isolated immature NP cells are not highly adhesive to tissue culture plastic or ECM proteins traditionally used to promote cell adhesion in vitro (e.g., collagen, fibronectin), we investigated NP cell attachment to those laminin isoforms shown to be expressed in situ (LM-511 (Chen et al., 2009), LM-332 (Fig. 1)). As shown in Fig. 2, freshly isolated NP cells were found to attach to LM-511 and LM-332CM substrates in significantly higher numbers than on other substrates $(p<0.05$ for all comparisons), with cell numbers approximately twice those of type II collagen substrates following either $30 \mathrm{~min}$ or 4 $\mathrm{h}$ of incubation. In contrast, LM-111 substrates supported significantly less attachment than other laminins at both time points (25-40\% of levels on LM-511 and LM-332 at both time points, $p<0.01$ ), with a trend towards less attachment compared to type II collagen $(p>0.05)$. Freshly isolated NP cells did not attach to fibronectin substrates in significant numbers $(<5 \%$ of levels on collagen for both time points), suggesting that expression of receptors that mediate NP cell attachment to fibronectin, which have been shown to be expressed at low levels in immature NP cells (Gilchrist et al., 2007), may have been affected by the cell isolation protocol.

\section{Differential adhesion of IVD cells on laminins}

Since in situ expression of laminin isoforms and laminin cell-surface receptors has been shown to be unique to the NP region (Nettles et al., 2004; Chen et al., 2006; Gilchrist et al., 2007; Chen et al., 2009), we examined whether cells from the AF region exhibited similar cell interactions with laminins as observed for NP cells. As shown in Fig. 3, NP cells were found to attach in significantly greater numbers to all laminin substrates as compared to AF cells. The difference in numbers of cells attaching to laminin between AF and NP cells was greatest on the LM-111 substrates, where NP cells attached in numbers 2.5 times higher than AF cells. The mean fold-differences between AF and NP cell types on LM-511 and LM-332CM substrates were less, with NP cells attaching in numbers approximately 1.3 times higher than AF cells.

\section{NP cell spreading on specific ECM ligands}

NP cells were found to spread rapidly on LM-511 and LM-332CM substrates, with cell projected areas after 1 hour of attachment two- to three-fold higher than cells on collagen, fibronectin, and LM-111 substrates (Fig. 4, left). A two-way ANOVA detected significant effects of substrate $(p<0.0001)$ and time $(p<0.005)$, with no evidence of an interaction between these factors $(p=0.93)$. Cell areas on LM-511 and LM-332CM substrates were significantly greater than on other substrates, with less spreading found on (in decreasing order) LM-111, fibronectin, and type II collagen substrates. Over a 4 h time course, cells on LM511 and LM-332CM spread at similar rates and appeared 


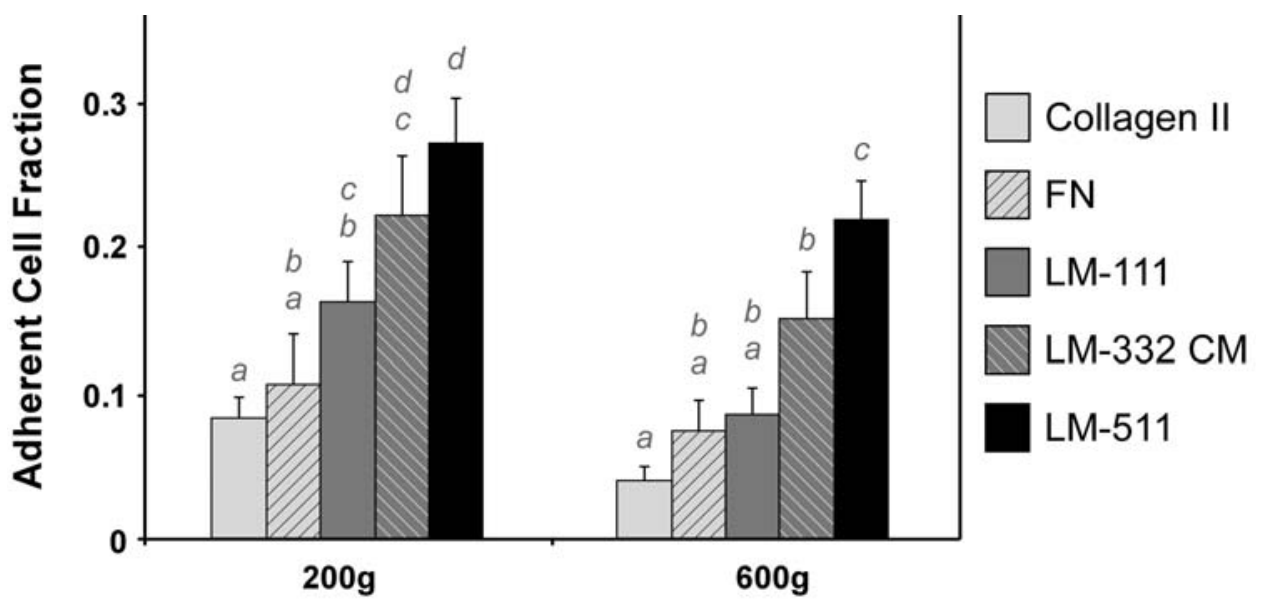

Applied Detachment Force

Fig. 5. NP cell relative attachment strength to ECM substrates. Fraction of NP cells (mean \pm SEM, $n=3$ independent experiments) remaining on ECM substrate surfaces following application of a normal centrifugal detachment force. For a given detachment force $(200 \mathrm{~g}, 600 \mathrm{~g})$, conditions not connected by same letter were statistically different (ANOVA, $p<0.05$ ).

to approach a maximum projected area of approximately $2,000 \mu \mathrm{m}^{2}$, whereas cells on LM-111, FN, and type II collagen slowly increased projected area over the time period (Fig. 4, left).

Analysis of cell shape factor during spreading (Fig. 4, right) indicated NP cells on LM-511 and LM-332CM, as well as on LM-111, had significantly lower shape factors (greater deviation from spherical shape, $p<0.001$ differences amongst substrates) than cells on collagen or fibronectin. Shape factor differences between substrates were apparent at $1 \mathrm{~h}$ and were maintained over the $4 \mathrm{~h}$ spreading experiment. Whereas NP cells on LM-111 substrates had intermediate projected areas in comparison to other substrates (greater than collagen/fibronectin but less than other laminin isoforms, Fig. 4, left), they exhibited shape factors comparable to the other laminin isoforms (Fig. 4, right). Corresponding to this finding, examination of NP cell morphology on LM-111 substrates revealed that cells typically exhibited a more elongated, spindle-shaped morphology in comparison to other substrates.

\section{NP cell attachment strength}

The attachment strength of NP cells was investigated by examining the ability of adherent NP cells to withstand a defined centrifugal detachment force. Following the application of a $200 \mathrm{~g}$ force, NP cells were found to remain adherent in significantly greater numbers on LM-511 and LM-332CM surfaces, with an adherent cell fraction approximately twice that of cells on collagen and fibronectin substrates (Fig. 5, left). The fraction of cells remaining on LM-111 surfaces was greater than on collagen surfaces, but less than on LM-511. A similar pattern was observed following application of a $600 \mathrm{~g}$ force (Fig. 5, right), with LM-511 having a significantly higher fraction than other substrates and collagen having the lowest adherent cell fraction. These results correspond closely with cell spreading results (Fig. 4), where LM-
511 and LM-332CM surfaces promoted significantly greater cell spreading rates as compared to collagen and fibronectin, and LM-111 spreading was generally less than other laminins but greater than collagen and fibronectin. These findings indicate NP cells form rapid, mechanically functional interactions with laminin isoforms LM-511 and LM-332, as compared to collagen and fibronectin ligands.

\section{Discussion}

Cell adhesion is a critical event affecting a variety of cellular behaviors, with specific ECM ligand-receptor interactions uniquely modulating cellular signaling, survival, growth, and phenotype. A major finding of this study is that the laminin isoforms LM-332 and LM-511 are preferred adhesive ligands for immature NP cells, in comparison to type II collagen, fibronectin, and another laminin isoform, LM-111. NP cells seeded on LM-511 and LM-332CM substrates attached rapidly and at higher numbers as compared to other substrates, and spread rapidly and to a greater extent in dynamic cell spreading experiments. Additionally, greater numbers of attached NP cells were found to resist a defined centrifugal detachment force when attached to these laminin isoforms, indicating that NP cells developed stronger mechanical interactions on these substrates.

NP cells exhibited very similar behaviors on LM-511 and LM-332CM substrates, with no differences detected in adhesion, spreading, shape, or relative attachment strength. It is notable that these two laminin isoforms are bound by an almost identical set of integrin receptors: $\alpha 6 \beta 4, \alpha 3 \beta 1$, and $\alpha 6 \beta 1$ (Miner and Yurchenco, 2004; Nishiuchi et al., 2006). This receptor set also corresponds with knowledge of integrin expression in immature NP cells, where high levels of integrin subunits $\alpha 6, \beta 1$, and $\beta 4$, as well as the non-integrin LM-511-specific receptor CD239, have been 
documented via flow cytometry and tissue immunostaining (Nettles et al., 2004; Chen et al., 2006; Gilchrist et al., 2007; Chen et al., 2009). Additionally, immunostaining of immature NP tissues has identified cell-associated localization of these laminin isoforms, with LM-332 (Fig. 1) and LM-511 ( $\alpha 5$ laminin subunit (Chen et al., 2009)) staining intensely in the NP region, in stark contrast to AF tissues. Together, these findings confirm that NP cell-matrix interactions and receptor expression observed in immature NP tissues translate into ex situ cell adhesion behaviors.

NP cell interactions with another laminin isoform, LM-111, were notably different, with NP cells exhibiting significantly lower levels of adhesion, spreading, and resistance to applied detachment forces on this isoform as compared to LM-332CM or LM-511 substrates. LM-111 is known to be a high affinity ligand for the $\alpha 6 \beta 1$ integrin, but not $\alpha 6 \beta 4$ or $\alpha 3 \beta 1$ integrins (Nishiuchi et al., 2006), and this difference in receptor binding may explain the measured differences in adhesion and spreading between laminin isoforms. That NP and AF cell numbers adhering to LM-111 differed further suggests that differences in $\alpha 6$ integrin subunit expression may exist between these cell types (Chen et al., 2006; Gilchrist et al., 2007). Reduced NP cell adhesion on LM-111 is also consistent with knowledge of IVD laminin isoform expression in situ, where the LM111 isoform (as identified by presence of the $\alpha 1$ chain) was not detected in porcine, rat, or human immature NP tissues (Chen et al., 2009).

Laminins (particularly the LM-511 and LM-332 isoforms) are important cell-adhesive ligands present in basement membranes and epithelial tissues (Miner and Yurchenco, 2004; Nguyen and Senior, 2006), and the finding that immature NP cells interact preferentially with these specific isoforms, as well as express receptors specific to these ligands (Chen et al., 2006; Gilchrist et al., 2007; Chen et al., 2009), highlights the epitheliallike phenotype of these notochordally-derived cells. The embryonic notochord has been described as a "primitive epithelia" based on cell-cell and cell-matrix adhesions identified histologically (Trout et al., 1982b; Hunter et al., 2003b). During IVD development, the notochord segments and expands to form the NP region of the IVD (Goto and Uhthoff, 1986; Rufai et al., 1995; Choi et al., 2008), and the notochordally-derived cells remaining in the NP have been shown to retain a number of epithelial characteristics (e.g., expression of cytokeratin intermediate filaments, cadherins, gap junctions (Gotz et al., 1995; Hunter et al., 2003b)). Our findings suggest that features of this epithelial-like phenotype are preserved in immature NP cells, with these cells exhibiting preferential interactions with basement membrane laminin ligands as compared to ligands more common to cartilaginous tissues (i.e., collagens, fibronectin).

Although LM-511 and LM-332 are two of the most common laminin isoforms present in a variety of mature tissues, studying cellular interactions with these isoforms is challenging, as it is difficult to obtain significant quantities of these ECM proteins in a purified form. These isoforms are difficult to extract from tissues (where they are present in low concentrations and are highly crosslinked within the ECM) or to purify from cell supernatant, and they are not easily produced recombinantly due to their large size and higher-order structure. In the present study, we used LM511 extracted from human placental laminin (Wondimu et al., 2006), and conditioned media containing LM-332 (Langhofer et al., 1993; Plopper et al., 1996), to study NP cell adhesion. LM-332CM is not purified laminin and may contain a variety of other constituents; however, previous work using LM-332CM immuno-depleted to remove LM-332 have shown that this isoform is critical for cell adhesion and spreading of keratinocytes (Hormia et al., 1995). In the present study, NP cell behaviors on LM332CM (attachment numbers, spreading, and attachment strength) were very similar to those on LM-511, and notably different from collagen, fibronectin, or LM-111 substrates. We hypothesize that NP cell interactions with LM-332 were key to observed behaviors on this substrate, since these two isoforms are known to share a very similar set of cell-matrix receptors (i.e., integrins $\alpha 6 \beta 4$, $\alpha 6 \beta 1$, and $\alpha 3 \beta 1)$.

The studies presented here utilize cells from immature porcine IVD tissues, which provide an abundant source of primary immature NP cells. Whether human NP cells (immature or mature) exhibit similar interactions with laminin is not known and should be the subject of further study. Immature human NP tissues and isolated cells have been shown to express laminins (LM-511) and laminin receptors (Chen et al., 2009), suggesting that some of the findings reported here may find application to the human. Furthermore, some phenotypic characteristics of human NP cells are maintained with maturity and aging (Minogue et al., 2010; Risbud et al., 2010) despite considerable changes in morphology and biosynthesis with maturation, aging, and degeneration (Trout et al., 1982a; Trout et al., 1982b; Roberts et al., 2006). Whether those characteristics of NP cells that regulate cell-laminin interactions are preserved with maturity and aging is not known, and merits future studies of human NP cell-laminin interactions as performed here.

The findings presented here may find application in the development of strategies that promote cellmediated regeneration of NP matrix in vitro or in vivo. Studies of adult NP cell or stem-like cell delivery to the disc space in vivo have shown that the incorporation of natural extracellular matrix components (e.g., collagen, atellocollagen, hyaluronan) in the cell delivery vehicle has positive effects for promoting cell retention and matrix regeneration (Crevensten et al., 2004; Gruber et al., 2006; Sakai et al., 2006; Kandel et al., 2008; Sakai, 2008). Those features of adult NP cells or stem-like cells that promote interactions with extracellular matrix components are unknown, although the preference of NP cells for laminin ligands observed here certainly suggests laminins could be one of several key extracellular matrix constituents that facilitate cell retention and potentially regulate cell behavior. Indeed, cell adhesion to laminin isoforms LM511 and LM-332 are known to direct a number of important cellular processes in other cell types, including promoting cell survival in response to environmental stress conditions such as nutrient deprivation and hypoxia (Gu et al., 2002), which may be relevant in the avascular disc. Additionally, these isoforms have been shown to maintain or direct 
cellular phenotype in a variety of tissues and cell types (Klees et al., 2005; Klees et al., 2007; Domogatskaya et al., 2008; Gao et al., 2008; Imanishi et al., 2010; Rodin et al., 2010). While the work presented in this study indicates NP cells preferentially adhere and interact with these laminin ligands, future work is necessary to assess the biological roles of these laminins in regulating NP cell behaviors.

\section{Conclusion}

In summary, this study highlights the unique interactions of immature NP cells with laminin ECM proteins. NP cells showed preferential attachment, spreading, and adhesion strength on substrates functionalized with two laminin isoforms, LM-511 and LM-332, as compared to another laminin isoform, LM-111, as well as to type II collagen and fibronectin, two other ECM constituents found in the disc. Additionally, NP cells were found to adhere in greater numbers to laminins as compared to AF cells. Together, these findings confirm that laminin protein and receptor expression identified in immature NP tissues translates into functional behaviors of isolated NP cells. This knowledge may be useful for promoting NP cell adhesion and (potentially) phenotype in vitro and in functionalized biomaterials for use in IVD tissue engineering applications.

\section{Acknowledgements}

This work was funded by NIH Grants EB002263, AR047442, AR057410, and T32 GM 8555 (for A.T.F.). The authors thank Steve Johnson, Liufang Jing, and Tiffany Chang for their assistance with tissue harvesting and cell adhesion experiments.

\section{References}

Aguiar DJ, Johnson SL, Oegema TR (1999) Notochordal cells interact with nucleus pulposus cells: Regulation of proteoglycan synthesis. Exp Cell Res 246: 129-137.

Bayliss MT, Johnstone B, O’Brien JP (1988) 1988 Volvo Award in Basic Science. Proteoglycan synthesis in the human intervertebral disc. Variation with age, region andpathology. Spine 13: 972-981.

Boos N, Weissbach S, Rohrbach H, Weiler C, Spratt KF, Nerlich AG (2002) Classification of age-related changes in lumbar intervertebral discs: 2002 Volvo Award in Basic Science. Spine 27: 2631-2644.

Boyd LM, Chen J, Kraus VB, Setton LA (2004) Conditioned medium differentially regulates matrix protein gene expression in cells of the intervertebral disc. Spine 29: 2217-2222.

Buckwalter JA (1995) Aging and degeneration of the human intervertebral disc. Spine 20: 1307-1314.

Cappello R, Bird JL, Pfeiffer D, Bayliss MT, Dudhia J (2006) Notochordal cells produce and assemble extracellular matrix in a distinct manner, which may be responsible for the maintenance of healthy nucleus pulposus. Spine 31: 873-882.
Chen J, Baer AE, Paik PY, Yan W, Setton LA (2002) Matrix protein gene expression in intervertebral disc cells subjected to altered osmolarity. Biochem Biophys Res Commun 293: 932-938.

Chen J, Jing L, Gilchrist CL, Richardson WJ, Fitch RD, Setton LA (2009) Expression of laminin isoforms, receptors and binding proteins unique to nucleus pulposus cells of immature intervertebral disc. Connect Tissue Res 50: 294-306.

Chen J, Yan W, Setton LA (2006) Molecular phenotypes of notochordal cells purified from immature nucleus pulposus. Eur Spine J 15 Suppl 3: S303-311.

Choi KS, Cohn MJ, Harfe BD (2008) Identification of nucleus pulposus precursor cells and notochordal remnants in the mouse: Implications for disk degeneration and chordoma formation. Dev Dyn 237: 3953-3958.

Colognato H, Yurchenco PD (2000) Form and function: The laminin family of heterotrimers. Dev Dyn 218: 213234.

Cotten A, Sakka M, Drizenko A, Clarisse J, Francke JP (1994) Antenatal differentiation of the human intervertebral disc. Surg Radiol Anat 16: 53-56.

Crevensten G, Walsh AJ, Ananthakrishnan D, Page P, Wahba GM, Lotz JC, Berven S (2004) Intervertebral disc cell therapy for regeneration: Mesenchymal stem cell implantation in rat intervertebral discs. Ann Biomed Eng 32: 430-434.

Domogatskaya A, Rodin S, Boutaud A, Tryggvason K (2008) Laminin-511 but not -332, -111, or -411 enables mouse embryonic stem cell self-renewal in vitro. Stem Cells 26: 2800-2809.

Engler A, Bacakova L, Newman C, Hategan A, Griffin M, Discher D (2004) Substrate compliance versus ligand density in cell on gel responses. Biophys J 86: 617-628.

Erwin WM, Ashman K, O’Donnel P, Inman RD (2006) Nucleus pulposus notochord cells secrete connective tissue growth factor and up-regulate proteoglycan expression by intervertebral disc chondrocytes. Arthritis Rheum 54: 3859-3867.

Erwin WM, Inman RD (2006) Notochord cells regulate intervertebral disc chondrocyte proteoglycan production and cell proliferation. Spine 31: 1094-1099.

Fujita N, Miyamoto T, Imai J, Hosogane N, Suzuki T, Yagi M, Morita K, Ninomiya K, Miyamoto K, Takaishi H, Matsumoto M, Morioka H, Yabe H, Chiba K, Watanabe S, Toyama Y, Suda T (2005) CD24 is expressed specifically in the nucleus pulposus of intervertebral discs. Biochem Biophys Res Commun 338: 1890-1896.

Gao J, DeRouen MC, Chen CH, Nguyen M, Nguyen NT, Ido H, Harada K, Sekiguchi K, Morgan BA, Miner JH, Oro AE, Marinkovich MP (2008) Laminin-511 is an epithelial message promoting dermal papilla development and function during early hair morphogenesis. Genes Dev 22: 2111-2124.

Gilchrist CL, Chen J, Richardson WJ, Loeser RF, Setton LA (2007) Functional integrin subunits regulating cell-matrix interactions in the intervertebral disc. J Orthop Res 25: 829-840.

Goto S, Uhthoff HK (1986) Notochord action on spinal development. A histologic and morphometric investigation. Acta Orthop Scand 57: 85-90. 
Gotz W, Kasper M, Fischer G, Herken R (1995) Intermediate filament typing of the human embryonic and fetal notochord. Cell Tissue Res 280: 455-462.

Gower WE, Pedrini V (1969) Age-related variations in protein polysaccharides from human nucleus pulposus, annulus fibrosus, and costal cartilage. J Bone Joint Surg Am 51: 1154-1162.

Gruber HE, Hoelscher GL, Leslie K, Ingram JA, Hanley EN, Jr. (2006) Three-dimensional culture of human disc cells within agarose or a collagen sponge: Assessment of proteoglycan production. Biomaterials 27: 371-376.

Gu J, Fujibayashi A, Yamada KM, Sekiguchi K (2002) Laminin-10/11 and fibronectin differentially prevent apoptosis induced by serum removal via phosphatidylinositol 3-kinase/Akt- and Mek1/Erkdependent pathways. J Biol Chem 277: 19922-19928.

Hormia M, Falk-Marzillier J, Plopper G, Tamura RN, Jones JC, Quaranta V (1995) Rapid spreading and mature hemidesmosome formation in HACAT keratinocytes induced by incubation with soluble laminin-5r. J Invest Dermatol 105: 557-561.

Hunter CJ, Matyas JR, Duncan NA (2003a) The notochordal cell in the nucleus pulposus: A review in the context of tissue engineering. Tissue Eng 9: 667-677.

Hunter CJ, Matyas JR, Duncan NA (2003b) The three-dimensional architecture of the notochordal nucleus pulposus: Novel observations on cell structures in the canine intervertebral disc. J Anat 202: 279-291.

Hynes RO (1992) Integrins: Versatility, modulation, and signaling in cell adhesion. Cell 69: 11-25.

Imanishi H, Tsuruta D, Tateishi C, Sugawara K, Paus R, Tsuji T, Ishii M, Ikeda K, Kunimoto H, Nakajima K, Jones JC, Kobayashi H (2010) Laminin-511, inducer of hair growth, is down-regulated and its suppressor in hair growth, laminin-332 up-regulated in chemotherapyinduced alopecia. J Dermatol Sci 58: 43-54.

Kandel R, Roberts S, Urban JP (2008) Tissue engineering and the intervertebral disc: The challenges. Eur Spine J 17 Suppl 4: 480-491.

Klaffky EJ, Gonzales IM, Sutherland AE (2006) Trophoblast cells exhibit differential responses to laminin isoforms. Dev Biol 292: 277-289.

Klees RF, Salasznyk RM, Kingsley K, Williams WA, Boskey A, Plopper GE (2005) Laminin-5 induces osteogenic gene expression in human mesenchymal stem cells through an ERK-dependent pathway. Mol Biol Cell 16: $881-890$.

Klees RF, Salasznyk RM, Vandenberg S, Bennett K, Plopper GE (2007) Laminin-5 activates extracellular matrix production and osteogenic gene focusing in human mesenchymal stem cells. Matrix Biol 26: 106-114.

Korecki CL, Taboas JM, Tuan RS, Iatridis JC (2010) Notochordal cell conditioned medium stimulates mesenchymal stem cell differentiation toward a young nucleus pulposus phenotype. Stem Cell Res Ther 1: 18.

Langhofer M, Hopkinson SB, Jones JC (1993) The matrix secreted by $804 \mathrm{G}$ cells contains laminin-related components that participate in hemidesmosome assembly in vitro. J Cell Sci 105: 753-764.

Lee CR, Sakai D, Nakai T, Toyama K, Mochida J, Alini M, Grad S (2007) A phenotypic comparison of intervertebral disc and articular cartilage cells in the rat. Eur Spine J 16: 2174-2185.

Loeser RF (1993) Integrin-mediated attachment of articular chondrocytes to extracellular matrix proteins. Arthritis Rheum 36: 1103-1110.

Lotz MM, Burdsal CA, Erickson HP, McClay DR (1989) Cell adhesion to fibronectin and tenascin: Quantitative measurements of initial binding and subsequent strengthening response. J Cell Biol 109: 17951805.

Miner JH, Yurchenco PD (2004) Laminin functions in tissue morphogenesis. Annu Rev Cell Dev Biol 20: 255 284.

Minogue BM, Richardson SM, Zeef LA, Freemont AJ, Hoyland JA (2010) Characterization of the human nucleus pulposus cell phenotype and evaluation of novel marker gene expression to define adult stem cell differentiation. Arthritis Rheum 62: 3695-3705.

Nettles DL, Richardson WJ, Setton LA (2004) Integrin expression in cells of the intervertebral disc. J Anat 204: 515-520.

Nguyen NM, Senior RM (2006) Laminin isoforms and lung development: All isoforms are not equal. Dev Biol 294: 271-279.

Nishiuchi R, Takagi J, Hayashi M, Ido H, Yagi Y, Sanzen N, Tsuji T, Yamada M, Sekiguchi K (2006) Ligand-binding specificities of laminin-binding integrins: A comprehensive survey of laminin-integrin interactions using recombinant $\alpha 3 \beta 1, \alpha 6 \beta 1, \alpha 7 \beta 1$ and $\alpha 6 \beta 4$ integrins. Matrix Biol 25:1 89-197.

Plopper G, Falk-Marzillier J, Glaser S, Fitchmun M, Giannelli G, Romano T, Jones JC, Quaranta V (1996) Changes in expression of monoclonal antibody epitopes on laminin-5r induced by cell contact. J Cell Sci 109: 19651973.

Plopper GE, Domanico SZ, Cirulli V, Kiosses WB, Quaranta V (1998) Migration of breast epithelial cells on laminin-5: Differential role of integrins in normal and transformed cell types. Breast Cancer Res Treat 51: 57-69.

Reyes CD, Garcia AJ (2003) A centrifugation cell adhesion assay for high-throughput screening of biomaterial surfaces. J Biomed Mater Res A 67: 328-333.

Risbud MV, Schaer TP, Shapiro IM (2010) Toward an understanding of the role of notochordal cells in the adult intervertebral disc: From discord to accord. Dev Dyn 239: 2141-2148.

Robbins PB, Lin Q, Goodnough JB, Tian H, Chen $\mathrm{X}$, Khavari PA (2001) In vivo restoration of laminin 5 $\beta 3$ expression and function in junctional epidermolysis bullosa. Proc Natl Acad Sci USA 98: 5193-5198.

Roberts S, Evans H, Trivedi J, Menage J (2006) Histology and pathology of the human intervertebral disc. J Bone Joint Surg Am 88 Suppl 2: 10-14.

Rodin S, Domogatskaya A, Strom S, Hansson EM, Chien KR, Inzunza J, Hovatta O, Tryggvason K, Boutaud A (2010) Long-term self-renewal of human pluripotent stem cells on human recombinant laminin-511. Nat Biotechnol 28: 611-615.

Rufai A, Benjamin M, Ralphs JR (1995) The development of fibrocartilage in the rat intervertebral disc. Anat Embryol (Berl) 192: 53-62. 
Sakai D (2008) Future perspectives of cell-based therapy for intervertebral disc disease. Eur Spine J 17 Suppl 4: 452-458.

Sakai D, Mochida J, Iwashina T, Watanabe T, Suyama K, Ando K, Hotta T (2006) Atelocollagen for culture of human nucleus pulposus cells forming nucleus pulposuslike tissue in vitro: Influence on the proliferation and proteoglycan production of HNPSV-1 cells. Biomaterials 27: 346-353.

Sakai D, Nakai T, Mochida J, Alini M, Grad S (2009) Differential phenotype of intervertebral disc cells: Microarray and immunohistochemical analysis of canine nucleus pulposus and anulus fibrosus. Spine (Phila $\mathrm{Pa}$ 1976) 34: 1448-1456.

Trout JJ, Buckwalter JA, Moore KC (1982a) Ultrastructure of the human intervertebral disc: II. Cells of the nucleus pulposus. Anat Rec 204: 307-314.

Trout JJ, Buckwalter JA, Moore KC, Landas SK (1982b) Ultrastructure of the human intervertebral disc. I. Changes in notochordal cells with age. Tissue Cell 14: 359-369.

Wondimu Z, Gorfu G, Kawataki T, Smirnov S, Yurchenco P, Tryggvason K, Patarroyo M, Herard AL, Pierrot D, Hinnrasky J, Kaplan H, Sheppard D, Puchelle E, Zahm JM (2006) Characterization of commercial laminin preparations from human placenta in comparison to recombinant laminins $2(\alpha 2 \beta 1 \gamma 1), 8(\alpha 4 \beta 1 \gamma 1), 10$ ( $\alpha 5 \beta 1 \gamma 1)$. Matrix Biol 25: 89-93.

\section{Discussion with Reviewers}

Reviewer I: Have you considered examining the gene expression profile of the immature NP cells that interact with these laminin isoforms? It would be interesting to see how these interactions influence the metabolism of the cell in terms of matrix synthesis etc.

Authors: We agree with the reviewer that this is a logical next step and are currently investigating NP cell responses to laminins for a follow-up study. There is little information in the literature regarding ECM production in response to culture on laminin ligands; however, several studies have documented the presence of cell-associated laminins in developing articular cartilage (Durr et al., 1996) and intervertebral disc (Hayes et al., 2001), suggesting a function in the production or assembly of cartilagenous tissues. Additionally, we have found that immature NP cells cultured on soft, laminin-functionalized gel substrates adhere readily but maintain a rounded cell shape (Gilchrist et al., 2009), which has been associated with enhanced proteoglycan synthesis in chondrocytes (e.g., Glowacki et al., 1983). We are currently investigating signaling and phenotypic responses to NP cell-laminin interactions as a follow-up to the work presented in this study.

Reviewer II: To confirm the preferential adhesion of NP cells to laminins in comparison to AF cells, the adhesion of AF cells to the other substrates, i.e., collagen and fibronectin, also needs to be shown. This is to test the possibility that AF cells generally show lower attachment to matrix molecules. Please comment.
Authors: We agree with the reviewer that attachment data for other substrates would be useful to compare overall levels of adhesion between cell types, and agree that this would add further information to this experiment. However, we note that the data in Fig. 3 does represent a direct adhesion comparison between cells from each region (same number of cells/well for each region allowed to attach to each substrate condition), and our findings directly support our conclusion that NP cells were more highly adhesive to laminins as compared to AF cells. We believe this to be relevant information independent of whether AF cells were more adhesive on other ECM substrates.

Reviewer II: It is evident from Fig. 3 that the attachment to LM-332CM and LM-511 in comparison to LM-111 is less increased than in the cells documented in Fig. 2. This may be a result of monolayer expansion and needs to be addressed.

Authors: We agree with the reviewer that the modest differences in relative attachment to laminin ligands between freshly isolated and monolayer cultured cells may be the result of different culture conditions, amongst other factors. Overall, we note that all measures of NP cell attachment to LM-511 or LM-332CM are statistically higher than attachment to LM-111, independent of cell culture; together we feel this data provides convincing evidence that NP cells will attach in higher numbers to LM-511 and LM-332CM.

Reviewer III: Please clarify the use of matrix proteins from different species.

Authors: The matrix proteins used in this study came from several different species (as identified in Methods, Cell Adhesion Experiments), and were selected depending on what was available commercially or through collaborators. It is true that differences may exist between species however, it is generally assumed that ECM proteins have a high degree of homology between mammalian species (e.g., human, rat, and bovine plasma fibronectin have $\sim 91$ $94 \%$ homology (Garcia-Pardo et al., 1985).

\section{Additional References}

Dürr J, Lammi P, Goodman SL, Aigner T, von der Mark K (1996) Identification and immunolocalization of laminin in cartilage. Exp Cell Res 222: 225-233.

Garcia-Pardo A, Pearlstein E, Frangione B (1985) Primary structure of human plasma fibronectin. Characterization of a 31,000 Dalton-fragment from the $\mathrm{COOH}$-terminal region containing a free sulfhydryl group and a fibrin binding site. J Biol Chem 260: 10320-10325.

Gilchrist CL, Darling EM, Chen J, Setton LA (2009) Immature nucleus pulposus cells self-assemble into cell clusters on soft laminin-rich substrates. Trans Orthop Res Soc 55: 1623.

Glowacki J, Trepman E, Folkman J (1983) Cell shape and phenotypic expression in chondrocytes. Proc Soc Exp Biol Med 172: 93-98.

Hayes AJ, Benjamin M, Ralphs JR (2001) Extracellular matrix in development of the intervertebral disc. Matrix Biol 20: 107-121. 